\title{
Detection of solar-like oscillations in the red giant star $\epsilon$ Ophiuchi by MOST spacebased photometry ${ }^{\star}$
}

\author{
C. Barban ${ }^{1,2}$, J. M. Matthews ${ }^{3}$, J. De Ridder ${ }^{2}$, F. Baudin ${ }^{4}$, R. Kuschnig ${ }^{3}$, A. Mazumdar ${ }^{5,2}$, R. Samadi ${ }^{1}$, \\ D. B. Guenther ${ }^{6}$, A. F. J. Moffat ${ }^{7}$, S. M. Rucinski ${ }^{8}$, D. Sasselov ${ }^{9}$, G. A. H. Walker ${ }^{3}$, and W. W. Weiss ${ }^{11}$ \\ 1 Observatoire de Paris, LESIA, CNRS UMR 8109, Place Jules Janssen, 92195 Meudon, France \\ e-mail: Caroline.Barban@obspm.fr \\ 2 Instituut voor Sterrenkunde, Katholieke Universiteit Leuven, Celestijnenlaan 200B, 3001 Heverlee, Belgium \\ 3 Department of Physics \& Astronomy, University of British Columbia, 6224 Agricultural Road, Vancouver V6T 1Z1, Canada \\ ${ }^{4}$ Institut d'Astrophysique Spatiale, CNRS/Université Paris XI UMR 8617, 91405 Orsay Cedex, France \\ 5 Astronomy Department, Yale University, PO Box 208101, New Haven, CT 06520-8101, USA \\ ${ }^{6}$ Department of Astronomy and Physics, St. Mary's University, Halifax, NS B3H 3C3, Canada \\ 7 Département de Physique, Université de Montréal, CP 6128, Succursale Centre-Ville, Montréal, QC H3C 3J7, Canada \\ 8 David Dunlap Observatory, University of Toronto, PO Box 360, Richmond Hill, ON L4C 4Y6, Canada \\ 9 Harvard-Smithsonian Center for Astrophysics, 60 Garden Street, Cambridge, MA 02138, USA \\ 10 Institut für Astronomie, Universität Wien, Türkenschanzstrasse 17, 1180 Wien, Austria
}

Received 8 November 2006 / Accepted 13 March 2007

\begin{abstract}
Context. Solar-like oscillations have been discovered in a few red giants, including $\epsilon$ Oph, through spectroscopy. Acoustic modes around $60 \mu \mathrm{Hz}$ were clearly seen in this star, but daily aliasing of the groundbased data made it impossible to unambiguously isolate the $p$-mode frequencies in the eigenspectrum, and hence the correct value of the large spacing, to asteroseismically constrain the mass of this pulsating star.

Aims. We obtained about 28 days of contiguous high-precision photometry of $\epsilon$ Oph in May-June 2005 with the MOST (Microvariability \& Oscillations of STars) satellite. The thorough time sampling removes the ambiguity of the frequency identifications based on the groundbased discovery data.

Methods. We identify equidistant peaks in the Fourier spectrum of the MOST photometry in the range where the $p$-modes were seen spectroscopically. Those peaks are searched by autocorrelation of the power spectrum to estimate the value of the large separation in the $p$-mode eigenspectrum. Having isolated the oscillation modes, we determine their mode parameters (frequency, amplitude and line width) by fitting the distribution of peaks to Lorentzian profiles.

Results. The clear series of equidistant peaks in the power spectrum, with amplitudes from about 30 to 130 ppm, are consistent with radial modes spaced by a mean value of $(5.3 \pm 0.1) \mu \mathrm{Hz}$. This large separation matches one of the two possibilities allowed by the groundbased observations thus constraining the stellar models to a much greater extent than previously possible. The line widths and Lorentzian fits indicate a rather short average mode lifetime: $\left(2.7_{-0.8}^{+0.6}\right)$ days.
\end{abstract}

Key words. stars: oscillations - stars: individual: $\epsilon$ Ophiuchi

\section{Introduction}

Oscillations similar to the $p$-modes (acoustic resonances) observed in the Sun are expected in other stars with an external convective envelope, where pulsations are stochastically excited by convective turbulence. It is therefore not surprising that such "solar-like" oscillations have been detected in red giants through radial velocity measurements (e.g., $\xi$ Hya, Frandsen et al. 2002; $\eta$ Ser, Barban et al. 2004; and the star of this paper, $\epsilon$ Oph, de Ridder et al. 2006). Red giant asteroseismology offers a unique opportunity to probe the interiors of evolved stars, but the limited time coverage and duty cycles of groundbased

^ Based on data from the MOST satellite, a Canadian Space Agency mission, jointly operated by Dynacon Inc., the University of Toronto Institute for Aerospace Studies, and the University of British Columbia, with the assistance of the University of Vienna. spectroscopy have made it difficult to identify $p$-mode frequencies unambiguously.

Solar-like oscillations have several typical characteristics:

- relatively low amplitudes;

- mode lifetimes that can be shorter than the observational time spans resulting in Fourier spectra with complicated peak profiles whose widths vary as the inverse of the lifetime; and - a comb of mode frequencies with approximately constant spacing (known as the "large separation") as expected from the asymptotic relation for $p$-modes of low degree and high radial order.

In the case of red giants, the theoretical calculations of Dziembowski et al. (2001) suggest that the eigenspectrum should be dominated by radial modes. Indeed, no clear evidence of nonradial modes was present in the radial velocity signals of the first red giant $p$-mode pulsators discovered. However, we note that Hekker et al. (2006) have argued that the line profile 
variations in these stars suggest the presence of nonradial modes, and Kallinger et al. (2007) has reported the detection of nonradial modes in MOST photometry of the red giant HD 20884.

$\epsilon$ Oph (G9.5III, $m_{v}=3.24$, HD 146791) was observed with the two echelle spectrographs CORALIE and ELODIE over 75 days in summer 2003 (de Ridder et al. 2006). A clear power excess is seen in the power spectrum of the radial velocity time series at frequencies near $60 \mu \mathrm{Hz}$. Despite two-site observations, the temporal duty cycle of the observations was still quite modest, making it impossible to distinguish clearly the true oscillation modes from the cycle/day aliases in the Fourier spectrum of the data. Thus, it was also impossible to reliably estimate mode parameters such as frequency, amplitude and lifetime, as well as the large separation of the eigenspectrum. For this last parameter, the range was narrowed to two possible values: around $5 \mu \mathrm{Hz}$ or $7 \mu \mathrm{Hz}$. Both values are consistent with shell hydrogen-burning stellar models, but lead to different possible masses for the star: $\sim 2 M_{\odot}$ or $\sim 2.8 M_{\odot}$. The masses of red giants are not well constrained by the classical stellar parameters, so there is great potential in the asteroseismic modeling of these stars through high$S / N$ measurements of oscillations with long nearly continuous time coverage. High $S / N$ and long time coverage are necessary to obtain good profiles of the $p$-mode peaks (and hence their frequencies and widths, and also mode identifications), while the high duty cycle is needed to overcome the ambiguities of aliasing. Observations from a spacebased instrument in the right orbit can satisfy all of these needs.

For these reasons, we decided to obtain photometry of $\epsilon$ Oph with the MOST (Microvariability \& Oscillations of STars) satellite (Walker et al. 2003; Matthews et al. 2004). MOST houses a $15-\mathrm{cm}$ telescope feeding a CCD photometer through a custom broadband optical filter. From its polar Sun-synchronous orbit, it is capable of observing certain bright stars for weeks at a time without interruption by Earth eclipses. For bright stars like $\epsilon$ Oph, MOST can obtain photometric precision as small as a few parts per million (ppm) in the expected frequency range for red giant $p$-modes.

\section{Observations and data analysis}

\subsection{Photometry}

$\epsilon$ Oph was monitored by MOST for about 28 contiguous days during 16 May-13 June 2005. The star was observed in MOST's Fabry Imaging mode (see Reegen et al. 2006), in which an extended image of the telescope pupil - illuminated by starlight is projected onto the Science CCD. The exposure time was $7.5 \mathrm{~s}$, sampled every $10 \mathrm{~s}$. Because of the position of $\epsilon$ Ophiuchi in the sky and the season of the observations, stray light due to scattered Earthshine became severe during the phase of the MOST satellite orbit over the Northern Hemisphere. We rejected those data so as not to degrade the overall quality of the photometry, resulting in a duty cycle of $46 \%$ but with no gaps longer than $87 \mathrm{mn}$ in the entire time series. The $46 \%$ duty cycle represents about half of every 101.4-min orbit of the satellite, so the aliases due to the gaps in the time series occur at spacings of about 14.2 cycles/day $(\sim 165 \mu \mathrm{Hz})$ instead of $1 \mathrm{c} / \mathrm{d}(\sim 11.6 \mu \mathrm{Hz})$.

The photometry reduction includes filtering of the stray light background modulated with satellite orbital parameters, filtering of long-term trends, and removal of obvious outliers. The standard deviation of the final light curve is $\sigma \sim 855 \mathrm{ppm}$, which includes intrinsic variations in the star itself. The light curve is shown in Fig. 1.

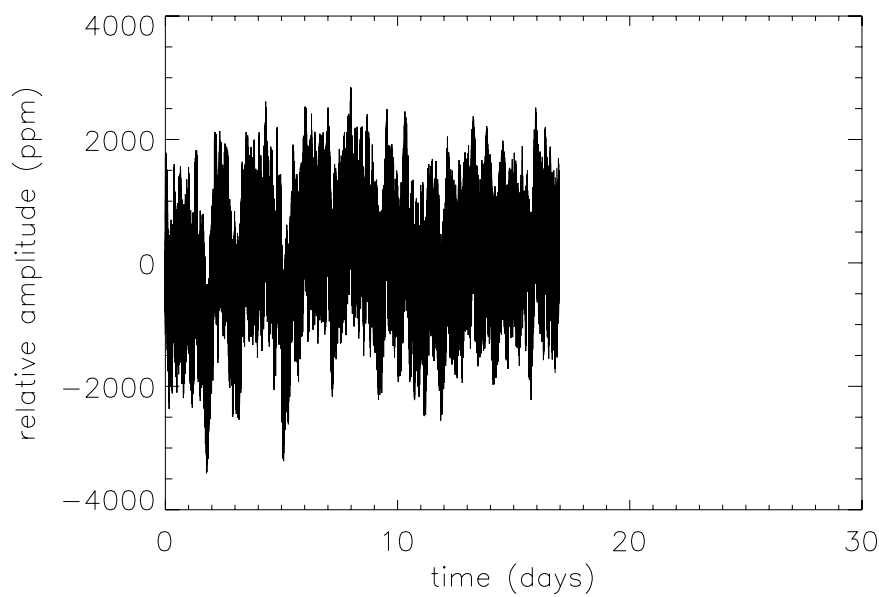

Fig. 1. Time series of the relative intensity variations of $\epsilon$ Oph obtained by MOST.

\subsection{Power spectrum analysis}

We searched for oscillation frequencies in the MOST photometry via a Discrete Fourier Transform (DFT) normalised by $2 / N$ where $N$ is the number of points in the time series. In this way, a sinusoid with an amplitude of $x$ will show a peak with power $x^{2}$ in units of $\mathrm{ppm}^{2}$. Frequencies in the DFT were sampled with a spacing of $1 / 4 T$, where $T$ is the total time span of the observations. The DFT was searched to high frequencies but no significant peaks are present beyond $100 \mu \mathrm{Hz}$ except those associated with the MOST satellite orbital frequency and its harmonics, and the $165-\mu \mathrm{Hz}$ aliases of the low-frequency power.

The power spectrum of the time series shown in Fig. 1 and the spectral window are presented in Fig. 2. We can see the satellite orbital aliases in the spectral window. Note that the aliases due to the gaps per satellite orbit in the MOST time series do not produce aliases in the frequency range where the $p$-modes are expected and found in these data; there are no aliases around $11 \mathrm{microHz}$ as for ground-based data. A clear series of apparently equidistant peaks is observed in the power spectrum, between about $25 \mu \mathrm{Hz}$ and $80 \mu \mathrm{Hz}$. This frequency range agrees with where the excess of power was seen by de Ridder et al. (2006) in their ground-based spectroscopy. The large peaks at frequencies below about $25 \mu \mathrm{Hz}$ are consistent with turbulence in the envelope of a red giant, and are not seen in the DFTs of the MOST guide stars in the $\epsilon$ Oph field. One of these is the relatively bright A star HD $146490(V=7.2$, A0IV), with 2-min binned point-to-point scatter of about $1000 \mathrm{ppm}$ (compared to $700 \mathrm{ppm}$ for $\epsilon$ Oph). The DFT of the light curve of HD 136490 is flat out to high frequencies and in particular shows no significant power in the frequency range where we identify oscillation peaks in $\epsilon$ Oph. The top panel of Fig. 2 shows the most prominent alias peaks due to the gaps in the photometry spaced by the MOST satellite orbital period of about $101.4 \mathrm{~min}$ (frequency of about $165 \mu \mathrm{Hz}$ ). The power at very low frequencies is consistent with turbulent variations in a red giant and may also contain some slow instrumental drifts. The aliases to those peaks are centred around $165 \mu \mathrm{Hz}$, where expected. There is distinctive excess power between about 25 and $75 \mu \mathrm{Hz}$ whose frequency range and other characteristics can be explained by solar-like p-mode oscillations of the star. The expected aliases to this envelope of peaks are found in the ranges $90-140 \mu \mathrm{Hz}$ and $190-240 \mu \mathrm{Hz}$, also where expected from the spectral window of the data.

We have computed a smoothed version of the power spectrum (Fig. 3) that clearly shows a series of approximately equally 

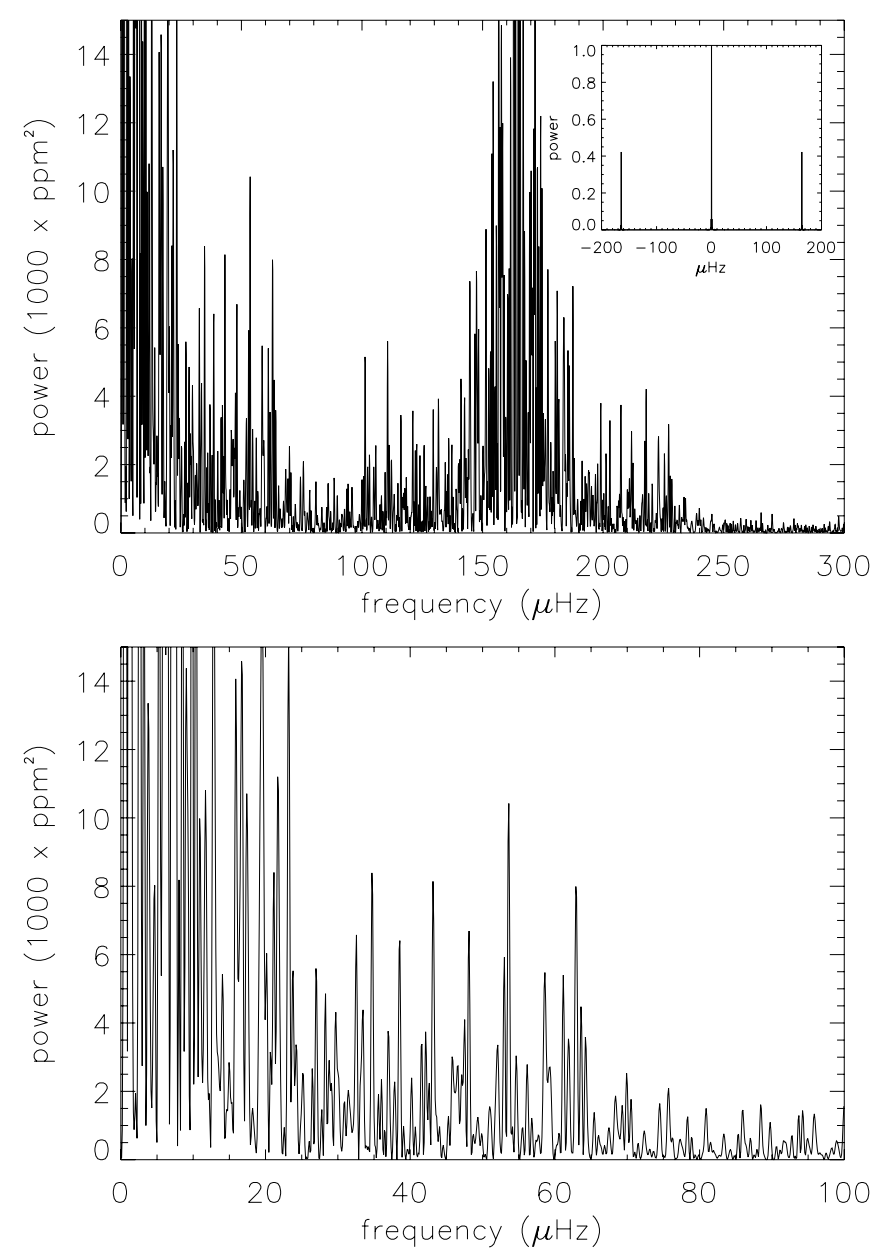

Fig. 2. Power spectrum of the $\epsilon$ Oph photometry for two different frequency ranges. The inset in the upper panel shows the spectral window.

wide peaks, with an average separation of about $5 \mu \mathrm{Hz}$. To better quantify this spacing, we searched for frequency equidistances (a characteristic of solar-like oscillations) by computing the autocorrelation of the power spectrum. All points in the power spectrum with a power greater than $3400 \mathrm{ppm}^{2}$ (corresponding to a $S / N \sim 3$, in amplitude) between 25 and $85 \mu \mathrm{Hz}$ have been used to reduce the effect of the noise in the autocorrelation. The noise was estimated by calculating the mean power between 78 and 90 microHz. The autocorrelation is shown in Fig. 4 and reveals two clear features around $5 \mu \mathrm{Hz}$ and $2 \times 5=10 \mu \mathrm{Hz}$. This is the unambiguous signature of a frequency spacing near $5 \mu \mathrm{Hz}$.

The width of the primary peak in the autocorrelation around $5 \mu \mathrm{Hz}$ as well as the shape of the smoothed power spectrum are both consistent with the $p$-mode power defined by broad Lorentzian envelopes. In Fig. 5, the time series has been divided into thirds and the power spectra of the three consecutive 9.3-day-long subsets are superposed. The changes in these three time intervals are substantial, yet not total, and are inconsistent with the low noise level of each subset. This supports the idea that the oscillations seen here are likely stochastic, with a mode lifetime shorter than about 10 days.

\section{Estimating $p$-mode parameters}

\subsection{Mode fitting model}

To estimate the parameters of stochastically excited modes, we have used a power spectrum fitting technique that has been

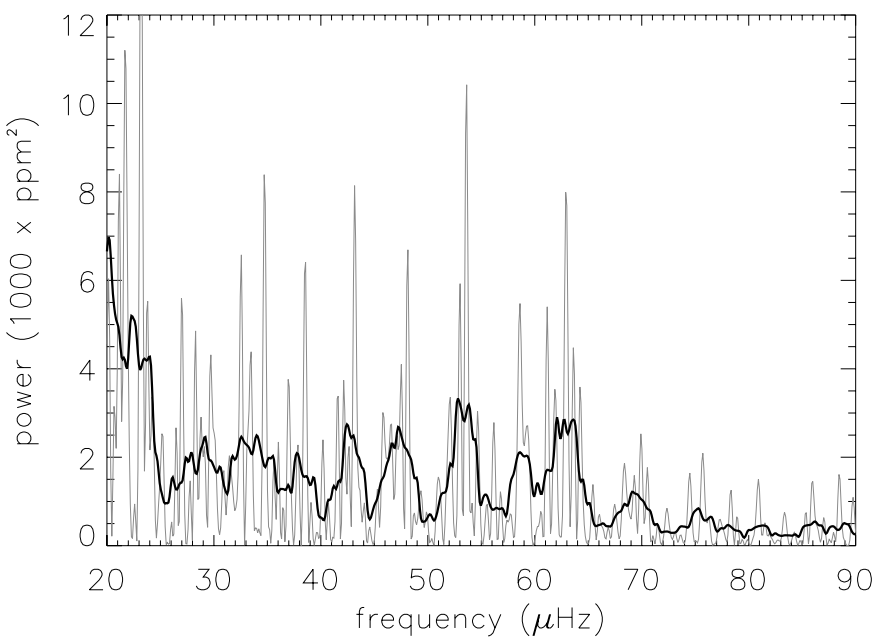

Fig. 3. Smoothed power spectrum of the $\epsilon$ Oph data (thick black solid curve). The light grey curve corresponds to the power spectrum shown in Fig. 2.

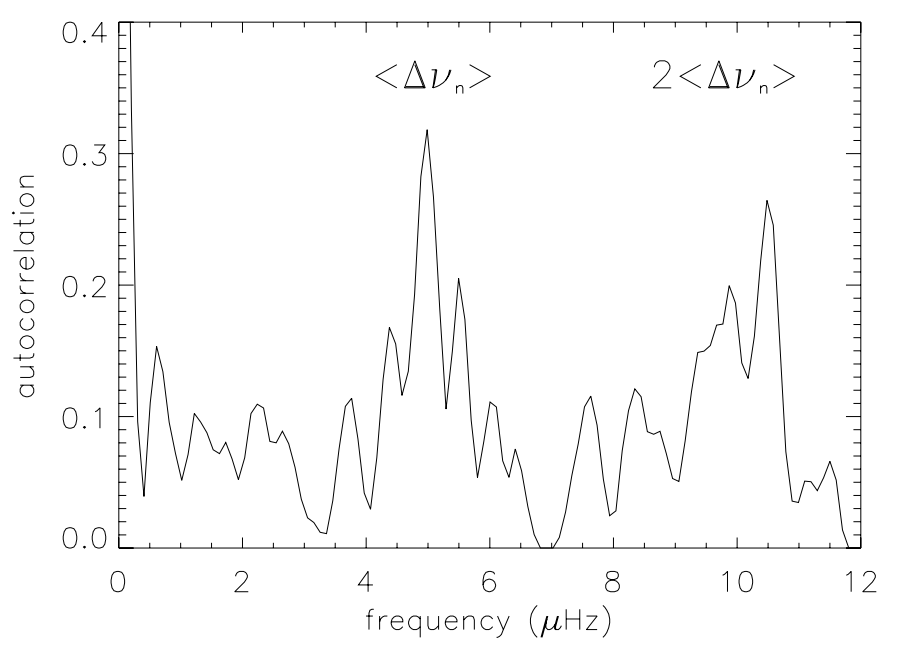

Fig. 4. Autocorrelation of the power spectrum. All points in the spectrum between 25 and $85 \mu \mathrm{Hz}$ with a power greater than $3400 \mathrm{ppm}^{2}$ (corresponding to a $S / N \sim 3$ ) have been used.

used succesfully in the solar case (Toutain \& Fröhlich 1992; Appourchaux et al. 1998).

Since solar-like oscillations are stochastically excited by convection, they can be modelled as randomly forced, damped, simple harmonic oscillators. The power spectrum of such oscillations is distributed around mean Lorentzian profiles and it has a $\chi^{2}$ probability distribution with two degrees of freedom. Following Toutain \& Appourchaux (1994), we have used a maximum likelihood technique to find the best fit between the model (Lorentzian) profiles and the observed power spectrum.

The modelled power spectrum for a series of $M$ oscillation modes, $P\left(v_{k}\right)$, is:

$P\left(v_{k}\right)=\sum_{n=1}^{M}\left(H_{n} \frac{1}{1+\left(\frac{2\left(v_{k}-v_{n}\right)}{\Gamma_{n}}\right)^{2}}\right)+B$

where $M$ is the number of oscillation modes, $H_{n}$ is the height of the Lorentzian profile, $v_{n}$ is the oscillation mode frequency and $\Gamma_{n}$ is the mode line width $(F W H M)$ with $\Gamma_{n}=1 /\left(\pi \tau_{n}\right), \tau_{n}$ being the mode lifetime. $B$ is the background noise in the power spectrum and has been assumed to be white in the frequency range used for the fit. 


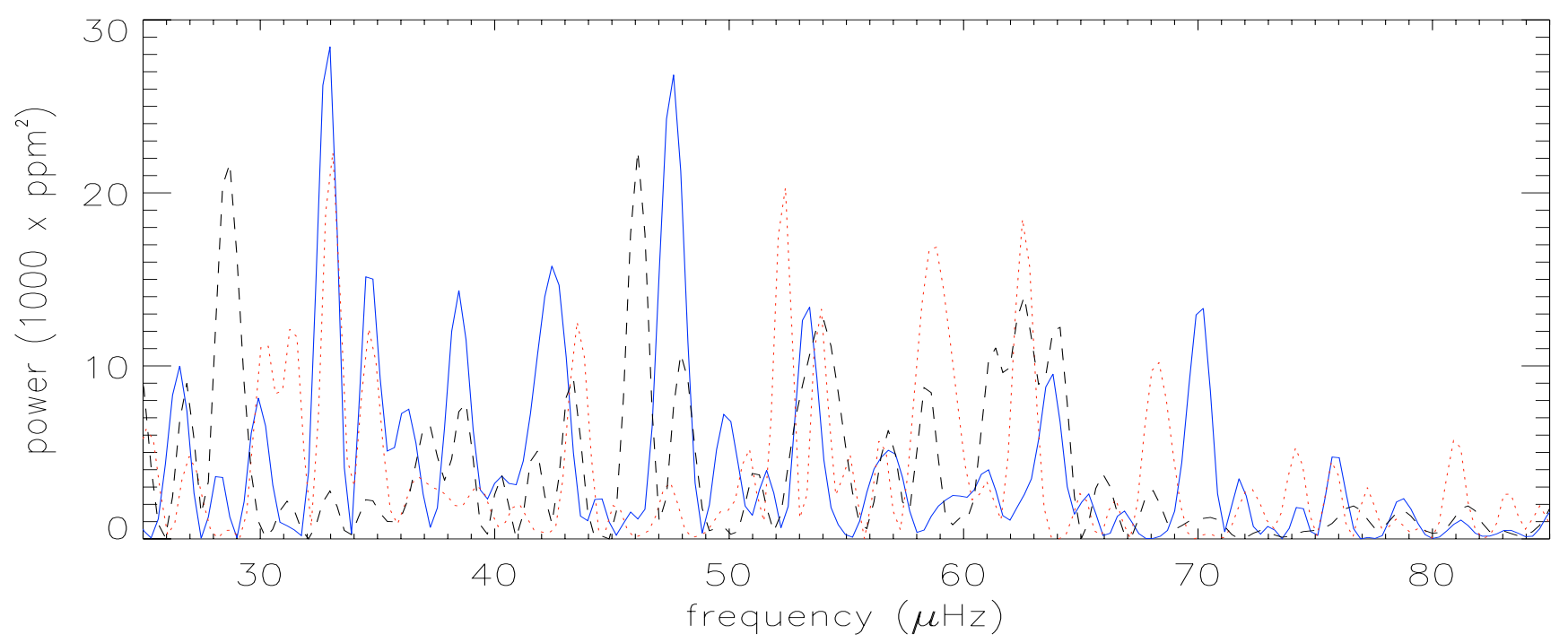

Fig. 5. The power spectra of three 9.3-day-long subsets of the MOST $\epsilon$ Oph light curve.

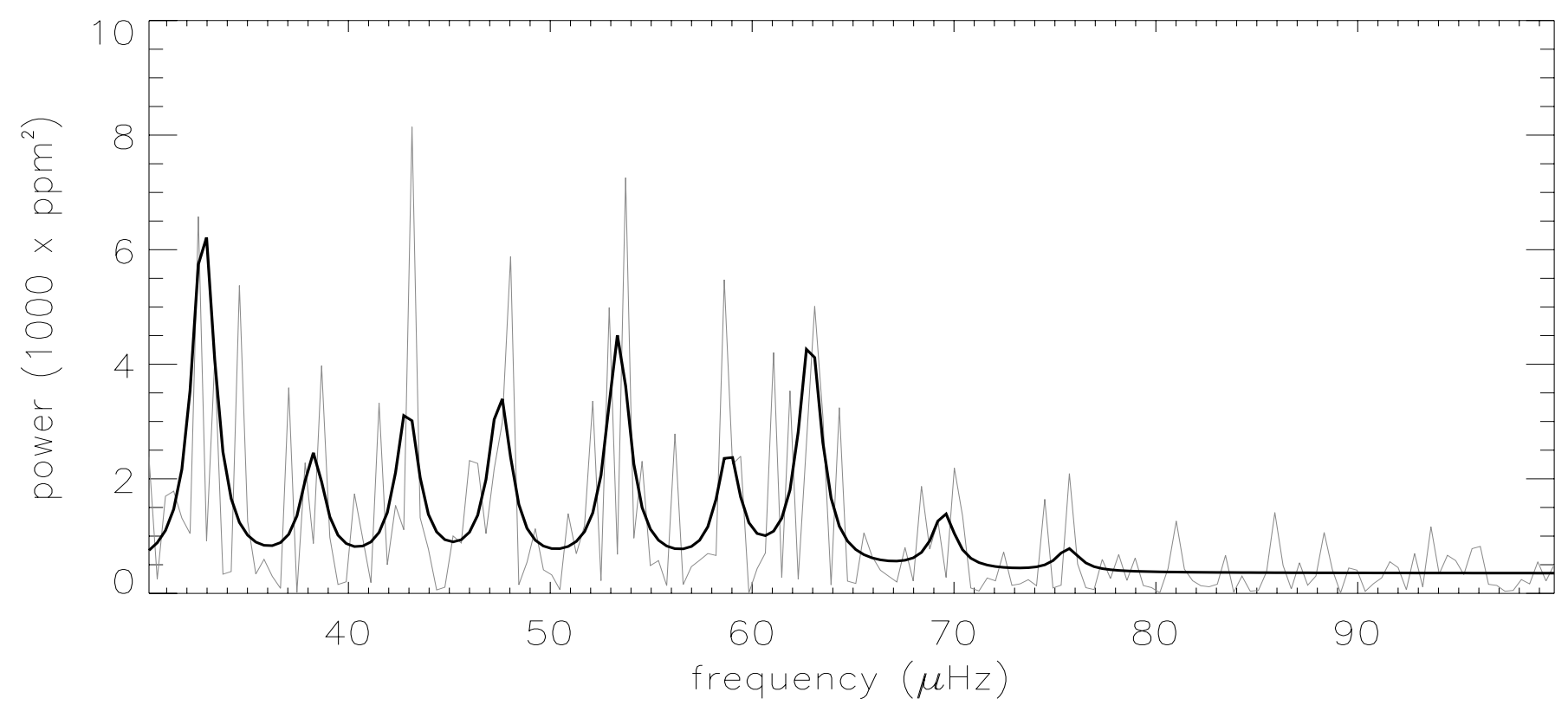

Fig. 6. Model fit (thick solid line) to the observed power spectrum (light gray line) assuming the same lifetime for all the oscillation modes.

The quantity that has been minimized is:

$L=\sum_{k=1}^{K}\left(\ln P\left(v_{k}\right)+\frac{P_{\mathrm{obs}}\left(v_{k}\right)}{P\left(v_{k}\right)}\right)$,

where $K$ is the number of bins; i.e., the number of Fourier frequencies.

The relation between the height of the Lorentzian profile, $H_{n}$, in the power spectrum computed from a Fourier Transform normalised by $2 / N$, and the mode amplitude in ppm, $a_{n}$, is:

$a_{n}^{2}=\pi \mathcal{H}_{n} \Gamma_{n}=\pi H_{n} \Gamma_{n} \frac{T}{4}$,

where $\mathcal{H}_{n}$ is the height of the Lorentzian profile in the power spectral density (see for example Baudin et al. 2005, for more details).

The mode-parameter $1 \sigma$ error bars are derived from the Hessian matrix. In the case of the amplitude $a_{n}$, as it is the product of two parameters which suffer cross-talk, their correlation has been taken into account to compute error bars.
No oversampling has been used in the computation of the power spectrum for the fitting procedure in order to minimize the interdependency of the points. This is why the power spectrum shown in Fig. 6 looks a little bit different from that in Fig. 2, where an oversampling factor of 4 was used.

\subsection{Mode fitting results}

Several strategies are possible when fitting modelled profiles to an observed spectrum. First, since the modes are quite close to each other, we have decided to fit all the modes simultaneously. Next, as each mode depends on several parameters, the number of fitted parameters is part of the fitting strategy, so we have started by fitting all the parameters of each mode. From Fig. 3, we can derive initial estimates for the central frequencies, amplitudes and lifetimes. The quality of our data is not sufficiently high to justify taking all mode parameters as free fitting parameters. When we attempted that, the fitting algorithm converged very easily to false minima, and we could not obtain reliable 
Table 1. Mode parameters for $\epsilon \mathrm{Oph}$. The results for the last two modes are written inside brackets to stress their low $S / N$. $1 \sigma$ errors are indicated. For the $S / N$ estimates, the signal is taken to be the height of the fitted Lorentzian profile and the noise to be a free parameter of the fitting process: $B$ in Eq. (1).

\begin{tabular}{|c|c|c|}
\hline$v_{n}(\mu \mathrm{Hz})$ & $a_{n}(\mathrm{ppm})$ & $S / N$ \\
\hline $32.8 \pm 0.5$ & $127_{-75}^{+93}$ & 17.5 \\
\hline $38.3 \pm 0.5$ & $71_{-49}^{+67}$ & 5.5 \\
\hline $42.9 \pm 0.4$ & $86_{-56}^{+74}$ & 8.0 \\
\hline $47.5 \pm 0.4$ & $89_{-56}^{+72}$ & 8.6 \\
\hline $53.4 \pm 0.4$ & $104_{-62}^{+78}$ & 11.6 \\
\hline $58.8 \pm 0.4$ & $72_{-49}^{+67^{\prime}}$ & 5.7 \\
\hline $62.9 \pm 0.4$ & $104_{-64}^{+80}$ & 11.7 \\
\hline$(69.5 \pm 0.8)$ & $\left(51_{-37}^{+52}\right)$ & 2.8 \\
\hline$\left(\begin{array}{ll}75.7 & \pm 0.5)\end{array}\right.$ & $\left(32_{-26}^{+42}\right)$ & 1.1 \\
\hline
\end{tabular}

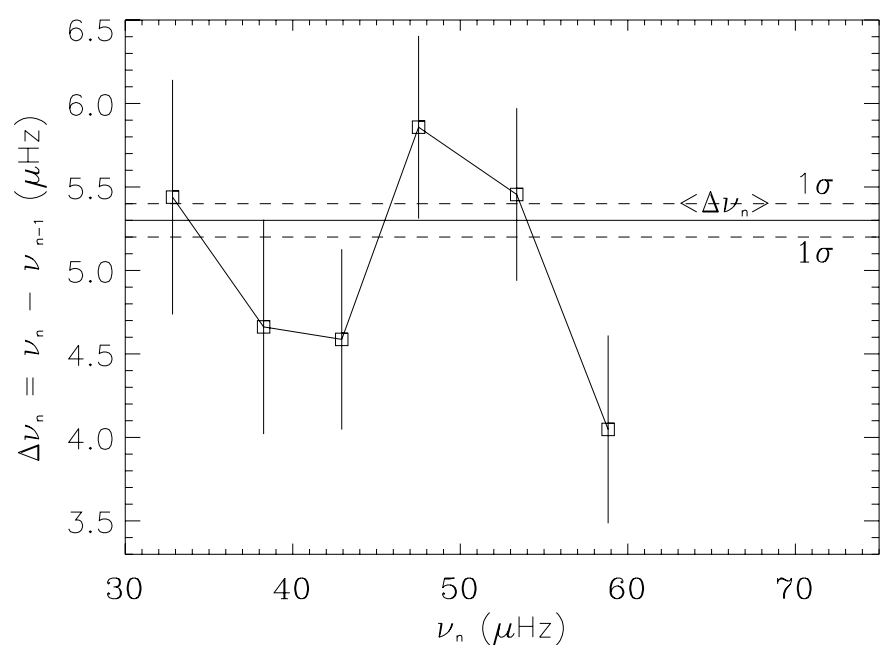

Fig. 7. Derived frequency differences in terms of a $p$-mode large separation, $\Delta v_{n}$, as a function of the mode frequency $v_{n}$. The solid line is the mean value of $\Delta v_{n}$ and the dashed lines indicate the corresponding $1 \sigma$ error.

results for a large number of modes. Since the apparently properly fitted modes had very similar width, we made the secondstep approximation that $\tau_{n}=\tau$ be constant. In this case, the fitting algorithm converges to a unique minimum, independent of the initial estimates of the parameters. Nine modes in total are properly fitted. The results of this fit between 30 and $80 \mu \mathrm{Hz}$ are shown in Fig. 6. The mode parameters are listed in Table 1. We stress that the last two modes have very low $S / N$ and that their mode parameters should be used with caution. We do not use these two modes in the following analysis.

The frequency differences from our model fit are shown in Fig. 7. The mean value of those frequency differences is derived by fitting a linear function to the measured frequencies assuming equidistant separation. A mean value of the frequency differences of $(5.3 \pm 0.1) \mu \mathrm{Hz}$ is found (consistent with our analysis in Sect. 2) which we attribute to the large separation of the $p$-modes in this star.

We have found a mode line width $(F W H M)$ of:

$\Gamma_{n}=\left(1.4_{-0.3}^{+0.4}\right) \mu \mathrm{Hz}$

corresponding to a mode lifetime of:

$\tau_{n}=\left(2.7_{-0.8}^{+0.6}\right)$ days.
The mode amplitudes $a_{n}$ (see Eq. (3)) range between about 30 and $130 \mathrm{ppm}$ (with large uncertainties; see Table 1 for the error bars).

\section{Conclusions}

The red giant star, $\epsilon$ Oph, has been observed for about 28 contiguous days by the Canadian satellite MOST. A series of peaks is seen in the power spectrum of the light curve between 25 and $80 \mu \mathrm{Hz}$. This frequency range corresponds to the frequency range where an excess power has been detected using spectroscopic measurements from the ground (de Ridder et al. 2006). The precision of the photometry and the high duty cycle and sampling of the MOST photometry allows one to obtain for the first time an estimate of the $p$-mode parameters for a red giant.

A clear signature of equidistant peaks is found, leading to a mean value of the large separation of $5.3 \pm 0.1 \mu \mathrm{Hz}$. This is in agreement with one of the two candidate separations $(4.8 \mu \mathrm{Hz})$ found by de Ridder et al. (2006) but which could not be identified due to the daily aliasing of their groundbased data. This value of about $5 \mu \mathrm{Hz}$ favours the low mass $\left(M \sim 2 M_{\odot}\right)$ shell hydrogenburning models presented by de Ridder et al. (2006), assuming that the observed modes are radial. A more detailed description of possible models, including core helium-burning models, will be presented in a forthcoming paper.

The detected equidistant peaks are consistent with 9 radial modes, and with the prediction of larger amplitudes for radial modes (Dziembowski et al. 2001) in red giants. While, in principle, it is conceivable that some or all of these modes could be nonradial, there is no significant evidence in the present data to either confirm or eliminate that possibility. However, the power spectrum strongly resembles the comb-like structure expected for radial modes, rather than a much denser spectrum of nonradial mixed modes that we expect for such evolved stars. We can rule out the possibility of successive $\ell=0$ and $\ell=1$ modes by the constraints on the luminosity and radius of this star which exclude a large $p$-mode spacing of $10 \mu \mathrm{Hz}$.

The frequencies, amplitudes and line widths of the 9 identified radial modes are estimated using a classical power spectrum fitting procedure. We find a mode line width $(F W H M)$ of $\left(1.4_{-0.3}^{+0.4}\right) \mu \mathrm{Hz}$ corresponding to a mode lifetime of only $\left(2.7_{-0.8}^{+0.6}\right)$ days. This value is similar to the one found for another red giant, $\xi$ Hya, by Stello et al. (2004, 2006). They found an average mode lifetime of 2 days, much shorter than the theoretical value of 15-20 days predicted by Houdek \& Gough (2002). Recently Kallinger et al. (2007) have claimed that oscillations discovered in MOST photometry of another red giant, HD 20884, are consistent with Houdek \& Gough's mode lifetime prediction of about 10 days for a star of its global parameters. For main sequence stars other than the Sun, the best observed estimate of mode lifetime has been obtained for $\alpha$ Cen A (G2V) by Fletcher et al. (2006). They found a value of $3.9 \pm 1.4$ days using fitting techniques similar to the one we use in this paper on WIRE space data. This value is in reasonable agreement with theoretical estimates (see Houdek 2006). There is also a mode lifetime determination for the companion of $\alpha$ Cen A, $\alpha$ Cen B, found to be similar to that in the Sun (between 1.9 and 3.3 days) (Kjeldsen et al. 2005).

Using appropriate values for the global characteristics of $\epsilon$ Oph $\left(L / L_{\odot}=59\right.$ and $T_{\text {eff }}=4900 \mathrm{~K}$; see de Ridder et al. 2006) and the mass appropriate to our $p$-mode identifications $(M=$ $2 M_{\odot}$ ), the predicted maximum mode amplitude is $\sim 50 \mathrm{ppm}$ according to the theory of Samadi et al. (2005). The mode 
amplitudes we obtain from the MOST data of $\epsilon$ Oph (while systematically higher than this prediction) agree with this value within $1 \sigma$. Few observational estimates of mode lifetimes and amplitudes exist to date because of the low frequency resolutions of most data sets and the ambiguities of aliasing due to gaps in those observations. This paper demonstrates the effectiveness of long continuous time coverage, currently possible from space. More (and more accurate) measurements of lifetimes and amplitudes will help to improve the treatment of convection in the stellar models, which is still uncertain (see Houdek 2006, for a recent review).

MOST has discovered other pulsating red giants (e.g., HD 20884; Kallinger et al. 2007) and is scheduled to monitor others to further investigate the issues of radial vs. nonradial modes, and mode lifetimes and amplitudes, in red giants. The MOST results presented here also demonstrate the promise of the next generation of space photometry missions, such as COROT (Baglin et al. 2003), whose extended time coverage and improved frequency resolution of red giant targets will represent another major advance in the field.

Acknowledgements. Part of this work was supported by the Research Fund of K. U. Leuven under grant GOA/2003/04 for C.B. and A.M. J.D.R. is a postdoctoral fellow of the Fund for Scientific Research, Flanders. R.K. is supported by the Canadian Space Agency (CSA). The Natural Sciences and Engineering Research Council of Canada supports the research of D.B.G., J.M.M., A.F.J.M., S.M.R. G.A.H.W.W.W. is supported by the Aeronautics and Space Agency of FFG and the Austrian Science Fund (FWF) P17580. FB thanks Thierry Toutain and Thierry Appourchaux for helpful discussions about mode fitting.

\section{References}

Appourchaux, T., Gizon, L., \& Rabello-Soares, M.-C. 1998, A\&AS, 132, 107 Baglin, A. 2003, Adv. Space Res., 31, 345

Barban, C., de Ridder, J., Mazumdar, A., et al. 2004, Proceedings of the SOHO 14 / GONG Workshop, Helio- and Asteroseismology: Towards a Golden Future, New Haven, Connecticut, USA, ed. D. Danesy, ESA SP-559, 113

Baudin, F., Samadi, R., Goupil, M.-J., et al. 2005, A\&A, 433, 349

De Ridder, J., Barban, C., Carrier, F., et al. 2006, A\&A, 448, 689

Dziembowski, W. A., Gough, D. O., Houdek, G., \& Sienkiewicz, R. 2001, MNRAS, 328, 601

Fletcher, S. T., Chaplin, W.J., Elsworth, Y., Schou, J., \& Buzasi, D. 2006, MNRAS, 371, 935

Frandsen, S., Carrier, F., Aerts, C., et al. 2002, A\&A, 394, L5

Kallinger, T., Guenther, B. D., Weiss, W. W., et al. 2007, ApJ, submitted

Kjeldsen, H., Bedding, T. R., Butler, R. P., et al. 2005, ApJ, 635, 1281

Hekker, S., Aerts, C., de Ridder, J., \& Carrier, F. 2006, A\&A, 458, 931

Houdek, G. 2006, Proceedings of SOHO 18/GONG 2006/HELAS I, Beyond the spherical Sun Sheffield, UK, ed. K. Fletcher, Scientific, ed. M. Thompson, published on CDROM, ESA SP-624, 28.1

Houdek, G., \& Gough, D. 2002, MNRAS, 336, L65

Matthews, J. M., Kusching, R., Guenther, D. B., et al. 2004, Nature, 430, 51

Reegen, P., Kallinger, T., Frast, D., et al. 2006, MNRAS, 367, 1417

Samadi, R., Goupil, M.-J., Alecian, E., et al. 2005, J. Astrophys. Astr., 26, 171

Stello, D., Kjeldsen, H., Bedding, T. R., et al. 2004, Sol. Phys., 220, 207

Stello, D., Stello, D., Kjeldsen, H., Bedding, T. R., \& Buzasi, D. 2006, A\&A, 448,709

Toutain, T., \& Appourchaux, T. 1994, A\&A, 289, 649

Toutain, T., \& Fröhlich, C. 1992, A\&A, 257, 287

Walker, G. A. H., Matthews, J. M., Kuschnig, R., et al. 2003, PASP, 115, 1023 